\title{
A numerical study of heat diffusion using the Lagrangian particle SPH method and the Eulerian Finite-Volume method: analysis of convergence, consistency and computational cost
}

\author{
C. A. D. Fraga Filho ${ }^{1,2}$, D. F. Pezzin ${ }^{1} \&$ J. T. A. Chacaltana ${ }^{1}$ \\ ${ }^{1}$ Laboratory of Simulation of Free Surface Flows, \\ Federal University of Espirito Santo, Brazil \\ ${ }^{2}$ Federal Institute of Espirito Santo, Brazil
}

\begin{abstract}
In this paper, the Lagrangian Smoothed Particle Hydrodynamics (SPH) method (with different combinations of smoothing functions/numbers of particles), the Eulerian Finite-Volume method using different refinements of the meshes and the Analytical method were applied for the study of heat diffusion. The numerical simulations by the SPH method have been performed using cubic spline, quartics and quintic spline kernels. The discretization of the domain has been affected by the use of $50 \times 50,60 \times 60,70 \times 70,80 \times 80$ or $90 \times 90$ particles. It has been noticed that the phenomenon of particle inconsistency and the consequent emergence of the largest temperature differences has been noticed, when compared with the analytical solution, near the bottom corners. The lowest differences have been obtained when the interpolation smoothing function degree and the number of particles used were the highest. For the Finite-Volume method, the largest differences between temperatures have been observed near the bottom corners, however they were lower than those found with the use of the SPH method. To obtain better results it is necessary to make boundary corrections. The computational cost of the SPH method was higher than the Finite-Volume method and increased as we increased the number of particles or the interpolation kernel degree.
\end{abstract}

Keywords: SPH method, heat diffusion boundaries, particles inconsistency, computational cost. 


\section{Introduction}

Smoothed Particle Hydrodynamics (SPH) is a Lagrangian particle method utilized in the solution of Partial Differential Equations (PDEs) traditionally solved by Eulerian methods using mesh and/or grids as Finite Volumes, Finite Differences and Finite Elements. The Eulerian methods are based on a properly pre-defined mesh (structured or unstructured). The governing equations for the physical problem is converted into a set of algebraic equations with unknowns to the nodal field variables.

The SPH method is based on the hypothesis that continuous domain can be divided into a finite number of particles that do not interact with one another, i.e. it is a model of a particle. The functions and their derivatives are approximated by radial functions, known as kernels interpolation. There is no use of meshes and stable numerical solutions to the partial differential equations obtained.

Heat diffusion is a widely studied problem in literature and different approaches to the application of SPH modeling method were performed. Cleary and Monaghan [1] have showed a little change compared with the standard modeling SPH, with a prediction of the heat flux at the boundaries. Jeong et al. [2] have performed the decomposition of the second order partial differential equations into two of first-order. Schwaiger [3] has presented an implicit formulation adjusted to model the heat diffusion with linear free surface conditions. Rook et al. [4] have showed explicit and implicit formulations using a Laplacian operator and the Crank Nicholson method, in a transient regime. $\mathrm{Xu}$ [5] has performed a comparative study, using an explicit numerical method that used a Laplacian operator, and the Finite Element Method (FEM), where it has been employed the LS - DYNA (software developed by Livermore Software Technology Corporation). Chen et al. [6] have showed the influence at the boundaries in the results obtained by the SPH and proposed a correction method for the interpolations in regions near the boundaries, known as Corrective Smoothed Particle method (CSPM).

The Finite Volume method and the SPH method, using a Laplacian operator, has been implemented in this work, to study the heat diffusion. Solutions were obtained to the coincident positions of the domain (points of the meshes and particles). The results found by the Eulerian method and by the Lagrangian method have been compared with the analytical solution of the partial differential equation of second order, that was used as reference for the analyzes. In this work, it has been demonstrated the Laplacian operator's employment's suitability in the SPH for modeling the heat diffusion. The influence of the kernel interpolation and the number of particles used in the inner regions of the domain is also shown. The inconsistency of the Lagrangian method in regions close to the boundaries is perceived by the emergence of the largest temperature differences at the lower contours of the flat plate studied. The best results has been obtained, with the use of $90 \times 90$ particles and the quintic kernel as interpolating function, showed the largest processing time. 


\section{Mathematical modeling}

\subsection{Heat diffusion equation}

Being that $\Omega \subset \mathfrak{R}^{\mathrm{n}}$ the space domain and $(0, \mathrm{t})$ the time domain, the equation that governs the energy transfer in the absence of pressure fields, is presented below, using the Einstein notation:

$$
\rho\left(\frac{\partial e}{\partial t}+u_{i} \frac{\partial e}{\partial x_{i}}\right)=\frac{\partial q_{i}}{\partial x_{i}}+S
$$

where $e$ is the specific energy, $\rho$ is the density, $t$ is the time, $\mathrm{u}_{\mathrm{i}}$ is the velocity, $\mathrm{x}_{\mathrm{i}}$ are the Cartesian coordinates, $\mathrm{q}_{\mathrm{i}}$ is the heat flux, $S$ is the heat source, $i$ refers to each of the directions.

In the absence of heat sources, the heat diffusion equation, derived of the eqn (1) is written according to the Lagrangian referential as follows:

$$
\frac{1}{\alpha} \frac{d T}{d t}=\left[\frac{\partial}{\partial x_{i}}\left(\frac{\partial T}{\partial x_{i}}\right)\right],
$$

where $T$ is the temperature and $\alpha$ is the thermal diffusivity.

\section{Numerical modeling}

\subsection{SPH formulation}

By the Taylor expansion, it is possible to determine the temperature value at a point $X^{\prime}=\left(x_{1}^{\prime}, x_{2}^{\prime}\right)$, around a fixed point $X=\left(x_{1}, x_{2}\right)$.

$$
\begin{aligned}
T\left(x_{1}^{\prime}, x_{2}^{\prime}\right) & =T\left(x_{1}, x_{2}\right)+\left.\left(x_{1}^{\prime}-x_{1}\right) \frac{\partial T}{\partial x_{1}}\right|_{\left(x_{1}, x_{2}\right)}+\left.\left(x_{2}^{\prime}-x_{2}\right) \frac{\partial T}{\partial x_{2}}\right|_{\left(x_{1}, x_{2}\right)}+ \\
+ & \frac{1}{2}\left(\left.\left(x_{1}^{\prime}-x_{1}\right)^{2} \frac{\partial^{2} T}{\partial x_{1}^{2}}\right|_{\left(x_{1}, x_{2}\right)}+\left.\left(x_{2}^{\prime}-x_{2}\right)^{2} \frac{\partial^{2} T}{\partial x_{2}^{2}}\right|_{\left(x_{1}, x_{2}\right)}\right)+ \\
& +\left.\left(x_{1}^{\prime}-x_{1}\right)\left(x_{2}^{\prime}-x_{2}\right) \frac{\partial^{2} T}{\partial x_{1} \partial x_{2}}\right|_{\left(x_{1}, x_{2}\right)}+R_{n}\left(x^{\prime}-x\right)
\end{aligned}
$$

where $R_{n}\left(x^{\prime}-x\right)$ is the remainder of the Taylor expansion.

For the obtaining of the approximation of the Laplacian of the temperature from the Taylor expression, we use the properties of anti-symmetry and non-standardization of the kernel gradient (Petronetto et al. [7]). The SPH expression for the Laplacian operator is: 
18 Advanced Computational Methods and Experiments in Heat Transfer XIII

$$
\left.\left(\frac{\partial^{2} T}{\partial x_{1}{ }^{2}}+\frac{\partial^{2} T}{\partial x_{2}{ }^{2}}\right)\right|_{\left(x_{1}, x_{2}\right)}=2 \sum_{b=1}^{n} \frac{m_{b}}{\rho_{b}}\left[T\left(X_{a}\right)-T\left(X_{b}\right)\right] \frac{\partial W(r, h)}{\partial r} \frac{1}{r_{a b}}
$$

where:

the subscripts $a$ e $b$ refer to the fixed particle and its neighbors, respectively.

$n$ is the number of the neighboring particles of the fixed particle;

$r$ is the radial direction in the polar coordinate system;

$r_{a b}=\left|X_{a}-X_{b}\right|$.

Different kernels, $W(r, h)$, are employed in the SPH method. Liu and Liu [8] have proposed the use of the cubic spline kernel, due to its desirable mathematical behavior as well as its derivatives. Other kernels are often used in the interpolations. Table 1 shows the kernels used in this work, all to a 2D domain.

Table 1: Interpolation kernels employed in the SPH method.

\begin{tabular}{|c|c|}
\hline Kernel & $W(r, h)\left(\mathrm{m}^{-2}\right)$ \\
\hline $\begin{array}{l}\text { Cubic spline } \\
\text { kernel (Liu and } \\
\text { Liu [8]) }\end{array}$ & $\frac{15}{7 \pi h^{2}}\left\{\begin{array}{cr}{\left[\frac{2}{3}-\left(\frac{\left|r-r_{b}\right|}{h}\right)^{2}+\frac{1}{2}\left(\frac{\left|r-r_{b}\right|}{h}\right)^{3}\right],} & 0 \leq\left|r-r_{b}\right| \leq h \\
{\left[\frac{1}{6}\left(2-\left(\frac{\left|r-r_{b}\right|}{h}\right)^{3}\right)\right],} & h<\left|r-r_{b}\right| \leq 2 h \\
0, & \text { in other case. }\end{array}\right.$ \\
\hline $\begin{array}{l}\text { Quartic Kernel } \\
\text { (Lucy [9]) }\end{array}$ & 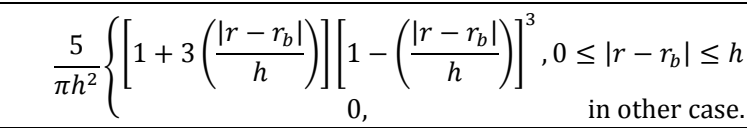 \\
\hline $\begin{array}{l}\text { Quartic Kernel } \\
\text { (Liu et al. [10]) }\end{array}$ & $\frac{15}{7 \pi h^{2}}\left\{\begin{array}{r}{\left[\frac{2}{3}-\frac{9}{8}\left(\frac{\left|r-r_{b}\right|}{h}\right)^{2}+\frac{19}{24}\left(\frac{\left|r-r_{b}\right|}{h}\right)^{3}-\right.} \\
\left.-\frac{5}{32}\left(\frac{\left|r-r_{b}\right|}{h}\right)^{3}\right], 0 \leq\left|r-r_{b}\right| \leq 2 h \\
0, \quad \text { in other case. }\end{array}\right.$ \\
\hline $\begin{array}{l}\text { Quintic Spline } \\
\text { Kernel (Morris et } \\
\text { al. [11]) }\end{array}$ & 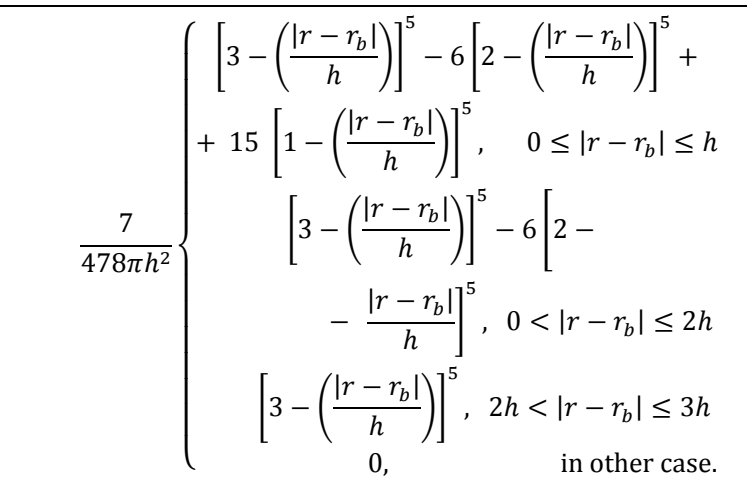 \\
\hline
\end{tabular}

$h$ is the support radius. 
The virtual particles, also called ghost particles, are generally employed in the defining of the boundary conditions of the SPH method (Monaghan [12]). In this work, prescribed temperature boundary conditions have been implemented using virtual particles with invariable temperatures during the whole time of the physical process.

\subsection{Consistency}

According to the Lax-Richtmeyer Equivalence Theorem, it is known that if a numerical model is stable, the convergence of the solution to the problem well-placed is determined by the consistency of the approximation function. In the SPH method, the consistency depends, besides the function approximation employed, the influence domain and the number/distribution of particles in its inside.

In regions where the influence domain is full, the consistencies of the orders 0 and 1 for the approximation function are guaranteed, due to the properties of the standardization and the symmetry of the kernel, presented respectively by the eqns (5) and (6).

$$
\begin{gathered}
\int W\left(X-X^{\prime}, h\right) d X^{\prime}=1, \\
\int\left(X-X^{\prime}\right) W\left(X-X^{\prime}, h\right) d X^{\prime}=0 .
\end{gathered}
$$

However, when the approach is extended to truncated regions by the boundaries, it is not guaranteed even the consistency of the order 0 for the approximation function.

Even though satisfying the kernel consistency conditions, it does not necessarily mean that the consistency of the SPH method is valid. It is fundamental the particle discretization domain analysis (known as particle consistency), given that the consistency of the method can be distorted in the discretization process.

The discrete counterparts for the kernel standardization and symmetry properties (that defines the consistencies of the $0^{\text {th }}$ and $1^{\text {st }}$ order) are as follows:

$$
\begin{gathered}
\sum_{b=1}^{n} W\left(r_{a}-r_{b}, h\right) \Delta V_{b}=1, \\
\sum_{b=1}^{n}\left(r_{a}-r_{b}\right) W\left(r_{a}-r_{b}, h\right) \Delta V_{b}=0 .
\end{gathered}
$$

where $\Delta V_{b}$ is the volume of each neighboring particle of the fixed particle and $n$ is the particle number at influence domain.

Figure 1 shows how the function approximation truncation occurs. The fixed particle is filled with black. 


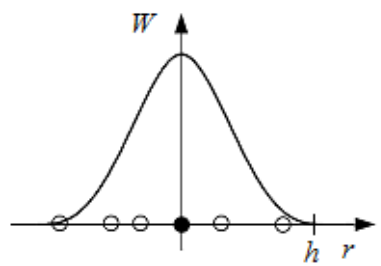

(a)

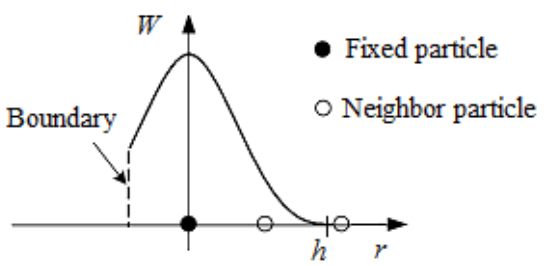

(b)

Figure 1: Influence domain. (a) Complete and (b) Incomplete. In (b) the fixed particle is near the contour and the kernel truncation occurs. Adapted from (Liu and Liu [8]).

In the border regions as shown in Figure 1(b), the kernel consistency is not guaranteed. There isn't a full influence domain and the kernel is truncated. This phenomenon is known as particles inconsistency and it leads to a lower accuracy in the interpolations (Liu and Liu [8]). Chen et al. [6] have proposed the application of the Corrected Smoothed Particle method (CSPM) aiming at correcting the particles inconsistency.

\subsection{Finite Volume formulation}

For the Finite Volume method, the property in the diffusive term has been defined as the temperature at the point $\mathrm{P}$ (center of the control volume).

From the governing eqn (1), without the existence of advection terms or heat sources, and applying the volume integral on its two sides, it's obtained:

$$
\int_{\forall} \frac{\partial P}{\partial t} d \forall=\int_{A} \alpha\left(\frac{\partial P}{\partial x_{i}}\right) \cdot n_{i} d A
$$

where $\forall$ is the volume, $A$ is the control volume surface and $n_{i}$ is the normal vector unit to the surface.

We arrive at the following numerical equation for the method:

$$
\begin{gathered}
P_{I, J}^{m+1}=P_{I, J}^{m}+\frac{\Delta t}{\Delta x_{1} \Delta x_{2}}\left(-\alpha\left(\frac{P_{I, J}^{m}-P_{I, J-1}^{m}}{\Delta x_{2}}\right) \Delta x_{1}+\right. \\
+\alpha\left(\frac{P_{I+1, J}^{m}-P_{I, J}^{m}}{\Delta x_{1}}\right) \Delta x_{2}+\alpha\left(\frac{P_{I, J+1}^{m}-P_{I, J}^{m}}{\Delta x_{2}}\right) \Delta x_{1}- \\
\left.-\alpha\left(\frac{P_{I, J}^{m}-P_{I-1, J}^{m}}{\Delta x_{1}}\right) \Delta x_{2}\right) .
\end{gathered}
$$

where:

the indices $m$ e $m+1$ represent the iterations related to the current time and advanced time, and the indices I and $\mathrm{J}$ represent, respectively, the coordinates of the control volume center; 
$\Delta x_{1}, \Delta x_{2}$ are the lengths of the sides of control volume in each Cartesian direction.

Ghost cells, or mirror cells, have been used for modeling the boundaries. Mathematically we have:

$$
-\left.\alpha \frac{\partial P}{\partial x_{2}}\right|_{I, J-1} ^{m} \Delta x_{1}=-2 \frac{\alpha}{\Delta x_{2}} \Delta x_{1}\left(P_{I, J}^{m}-P_{I, J-1}^{m}\right) .
$$

\section{Numerical simulations}

\subsection{Problem description}

The heat diffusion in a homogeneous flat plate with prescribed boundary conditions (Dirichlet boundary conditions) was studied. The dimensions and boundaries of the plate are shown in Figure 2. The whole domain has the temperature $\mathrm{T}_{0}$ in the initial time, i.e. the initial temperature is uniform.

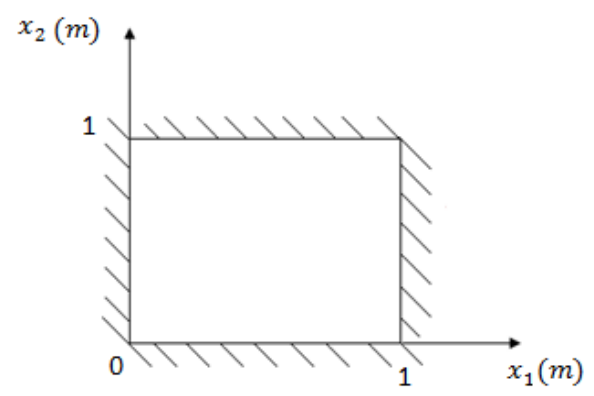

Figure 2: Homogeneous flat plate with initial and boundary conditions.

The initial condition is:

$T\left(x_{1}, x_{2}\right)=T_{0},\left(0<x_{1}<1\right.$ e $\left.0<x_{2}<1\right)$,

The boundary conditions are:

$$
\begin{array}{ll}
T\left(0, x_{2}\right)=0^{\circ} \mathrm{C} & \left(0 \leq x_{2} \leq 1\right), \\
T\left(1, x_{2}\right)=0^{\circ} \mathrm{C} & \left(0 \leq x_{2} \leq 1\right), \\
T\left(x_{1}, 0\right)=100^{\circ} \mathrm{C} & \left(0<x_{1}<1\right), \\
T\left(x_{1}, 1\right)=0^{\circ} \mathrm{C} & \left(0<x_{1}<1\right) . \\
\text { At steady state, eqn (2) becomes: }
\end{array}
$$

$$
\frac{\partial^{2} T}{\partial x_{1}^{2}}+\frac{\partial^{2} T}{\partial x_{2}^{2}}=0
$$

For the simulations using the Lagrangian method, the particles masses centers have been placed in the domain, separated by a distance $d x=d y=1 / n_{p}$, which $n_{p}$ is the number of particles on each side of the plate. The particles 
positions did not change with time. Five different values of $n_{p}(2500,3600$, 4900, 6400 and 8100) have been employed.

In the defining of the boundaries, virtual particles have been fixed on the side borders of the flat plate at a ratio of 02 virtual particles for each real particle of the domain. The physical properties and the support radius $(h)$ have been allocated for all particles. The thermal diffusivity was defined as $\alpha=1.0 \mathrm{~m}^{2} / \mathrm{s}$, with $\mathrm{k}=1.0 \mathrm{~J} / \mathrm{s}^{\circ} \mathrm{C} \mathrm{m} ; \rho=1.0 \mathrm{~kg} / \mathrm{m}^{3}$ e, $c_{v}=1.0 \mathrm{~J} / \mathrm{kg}^{\circ} \mathrm{C}$, held constant for all simulations. The time-step for all numerical simulations of this work was $1.10^{-5} \mathrm{~s}$. Virtual particles did not suffer interpolations over time to predict their temperatures, because they were invariable due to the Dirichlet boundary condition.

The calculation of the temperature Laplacian for each iteration was done explicitly by solving the following equation:

$$
\left(\frac{\partial^{2} T}{\partial x_{1}{ }^{2}}+\frac{\partial^{2} T}{\partial x_{2}{ }^{2}}\right)^{m}=2 \sum_{b=1}^{n} \frac{m_{b}}{\rho_{b}}\left[T\left(X_{a}\right)^{m}-T\left(X_{b}\right)^{m}\right] \frac{\partial W(r, h)}{\partial r} \frac{1}{r_{a b}} .
$$

In the Finite Volume method, five different types of uniform, regular and structured grids with $2500,3600,4900,6400$ and 8100 control volumes have been defined. The control volumes centers were coincident with the positions of the arranged particles in the SPH method.

The forecast temperatures for particles or control volumes was performed by the Euler numerical integration method.

$$
T^{m+1}=T^{m}+\left(\frac{\partial^{2} \mathrm{~T}}{\partial \mathrm{x}^{2}}+\frac{\partial^{2} \mathrm{~T}}{\partial \mathrm{y}^{2}}\right)^{m} \Delta t .
$$

where $\Delta t$ is the time-step.

Obtaining a difference between the temperatures for each particle/control volume center from at least $1.10-6^{\circ} \mathrm{C}$ between two successive iterations $\left(\left|T^{m+1}-T^{m}\right| \leq 1 \times 10^{-6^{\circ} \mathrm{C}}\right)$ was the criterion adopted for the end of the simulations.

Pletcher et al. [13] has employed the separation technique of the variables to obtain the solution by series for the eqn (12), that is:

$$
T\left(x_{1}, x_{2}\right)=\sum_{n=1}^{\infty} A_{n} \sin \left(n \pi x_{1}\right) \sinh \left[n \pi\left(x_{2}-1\right)\right] .
$$

where:

$$
A_{n}=\frac{2 T_{S}}{n \pi} \frac{\left[(-1)^{n}-1\right]}{\sinh (n \pi)}
$$

$T_{S}=T\left(x_{1}, 0\right)$ is the temperature at the lower boundary.

The results obtained by the SPH and Finite Volume methods have been compared with the latter solution. 


\section{Results and discussion}

The SPH simulations have showed that the number of particles employed in discretization of the domain had a significant influence on the solution. Another variable which was equally important was the kernel utilized. The largest differences between temperatures have been observed near the lower boundaries of the plate, regions where influence domains weren't complete, leading to a less accurate interpolation of the kernel gradient. By increasing the number of particles in the domain, the distances between them decreased, which has resulted in an elevation of the temperature gradients on those corners, for all kernels which have been utilized. However, when more particles have been added to the domain, it has been observed that the relative errors (obtained by comparing between the SPH results and analytical), despite being greater in magnitude, it has been restricted to a smaller number of special positions, close to the lower boundaries. It has been found that with the increasing number of particles, there has been a reduction of the temperature gradients in the inner regions. In a few positions the lower corners temperatures have become higher.

Regarding the interpolation functions employed, there was an influence on the kernel degree distributions of temperatures obtained. The smaller temperature differences in regions close to the boundaries have been observed on the highest degree kernel utilized (quintic spline), except for the distribution with 3600 particles. As shown by Chen et al. [6], the reduction of these differences between temperatures can be achieved by correcting of the interpolations obtained by the SPH, with the use of the Corrective Smoothed Particle method (CSPM).

For the Finite Volume method, it has been verified the existence of a relationship between the number of control volumes and the relative errors (encountered when temperatures were compared to those obtained by the Analytical method). The mesh refinement, starting from a certain number of volume elements (3600) only resulted in increased divergence between the methods, results in placed points at the bottom corners. This trend has been justified by the existence of strong temperature gradients in those positions and because the field is treated as constant, i.e. there isn't employment of any interpolating function.

The largest mesh refinement does not imply obtaining better results. The relative error showed an improvement of the mesh with $2500\left(2.28^{\circ} \mathrm{C}\right)$ for the mesh with 3600 control volumes $\left(2.20^{\circ} \mathrm{C}\right)$, which is ideal for the proposed problem. For meshes with 4900, 6400 and 8100 volumes, the relative error was higher, due to the strong gradient in the lower corners of the domain. Thus, the largest mesh refinement does not imply obtaining better results. The improvement of the solution can be achieved by the implementation of interpolators which soften the high temperature gradients.

The larger relative errors found by employing the two methods are shown in Figure 3. 


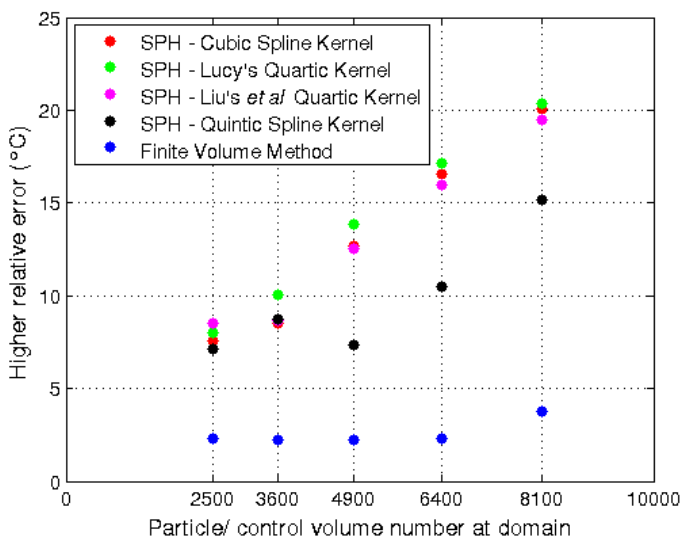

Figure 3: Behavior of the higher relative error for different methods adopted.

Serial codes developed in the FORTRAN Language have been used in the simulations. Data processing was performed using an Intel Core I5 $1^{\text {st }}$ generation processor. The physical time to the occurrence of heat diffusion from a transient regime until achieve steady state, with the achievement of the defined accuracy, was contained in the range of 0.43124 and 0.45366 s for the SPH method and between 0.45530 and 0.45539 s for the Finite Volume method, which has showed a good agreement between the results of both methods. The processing time has been measured for all simulations.

Figure 4 shows the CPU used time for the Eulerian and Lagrangian methods, with different numbers of control volumes or kernel combinations/particle numbers, respectively.

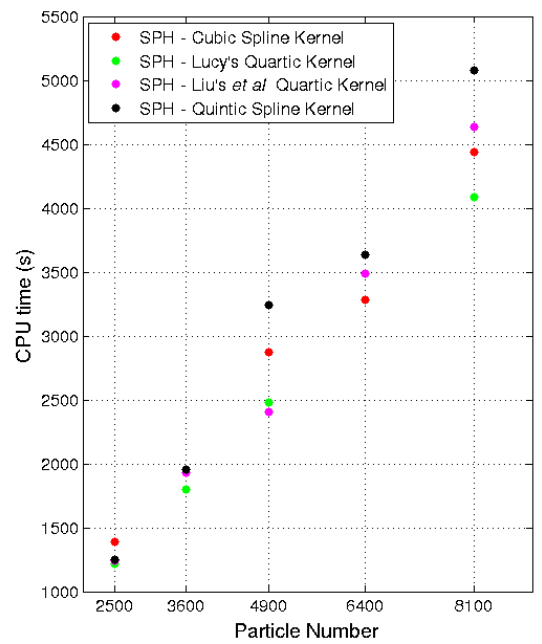

(a)

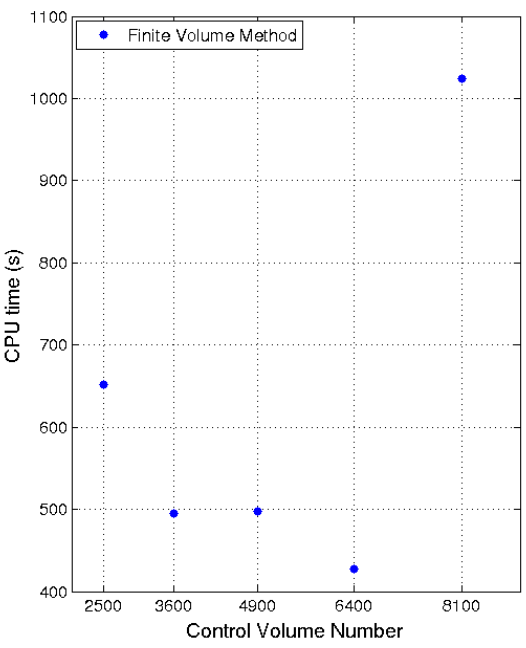

(b)

Figure 4: CPU time. (a) SPH and (b) Finite Volume method. 
In Finite Volume, the processing time has been shorter than those which has been observed through the SPH. For this method, it has been observed that with the increased of the particle number at domain there was also a processing time increase. For different kernels utilized, the one which presented a smaller computational cost in most of the simulations has been the Lucy's quartic kernel and the one which has showed the largest processing time, regardless of the initial particles arrangement in the field, was the quintic spline kernel.

\section{Conclusions}

The solutions provided by the SPH method have had different behaviors in two regions (near and far boundaries). In the inner regions of homogeneous flat plate, it has been observed that there is a concordance between the temperatures achieved by all methods (Lagrangian, Euler and Analytical) for all particles numbers and interpolation kernels. In regions near the boundaries, the SPH solution that have had the smallest deviations from the analytical solution was observed when it has been used the quintic spline kernel. On these regions, as there were an increase of the numbers of particles, there was a greater agreement with the analytical solution when considering all the studied domain, although the temperature gradients are larger near singular points in the corners of the plate, where there were larger relative errors.

For internal regions of the domain, the Laplacian of the temperature, which defines the heat diffusion equation in steady state, could be well approximated by the SPH Laplacian operator, which uses the kernel gradient in its formulation, since the discretization of the domain and the kernel interpolation were selected properly. In the case of this study, the best results have been observed when it has been used the 8100 particles and the quintic spline kernel as smoothed function.

In regions near the boundaries, we have observed the problem known as particles inconsistency, characterized by the truncation of the kernel near those regions. Consequently, the interpolation kernel gradient has been less precise, leading to larger relative errors. As a solution to this phenomenon Chen et al. [6] have proposed a correction method known as CSPM.

In the Finite Volume method, the largest relative errors has occurred in regions close to the lower boundary, in smaller magnitudes than those found for the SPH method. Simply refining the mesh has not led to better results, interpolators which soften the temperature gradient should be implemented.

The computational cost of the SPH method has been higher than that observed for the Eulerian Finite Volume method. Increasing the number of particles in the domain has impacted the time processing negatively. The use of higher degrees kernels has affected the simulation time adversely.

\section{References}

[1] Cleary, P. W. \& Monaghan J. J. Conduction Modelling Using Smoothed Particle Hydrodynamics. Journal of Computational Physics, 148: 227-264, 1999. 
[2] Jeong, J. H., Jhon M. S., Halow J. S. \& van Osdol J. Smoothed Particle Hydrodynamics: Applications to Heat Conduction. Computer Physics Communications, 153: 71-84, 2003.

[3] Schwaiger, H. F. An implicit corrected SPH formulation for thermal diffusion with linear free surface boundary conditions. International Journal for Numerical Methods in Engineering, 75: 647-671, 2008.

[4] Rook, R., Yildiz M. \& Dost S. Modeling Transient Heat Transfer Using SPH and Implicit Time Integration. Numerical Heat Transfer, Part B: Fundamentals, 51:1, 1-23, 2007.

[5] Xu, Jingxiao. Heat Transfer with Explicit SPH Method in LS-DYNA. Proceedings of the $12^{\text {th }}$ International LS-DYNA Users Conference. Computer Technologies. Detroit, USA, 2012.

[6] Chen, J. K., Beraun, J. E. \& Carney, T. C. A Corrective Smoothed Patricle Method for Boundary Value Problems in Heat Conduction. International Journal for Numerical Methods in Engineering, 46: 231-252, 1999.

[7] Petronetto, F., Paiva, A., Lage M., Tavares, G., Lopes, H. \& Lewiner T. Meshless Helmholtz-Hodge Decomposition. IEEE Transactions on Visualization and Computer Graphics, 16(2): 338-349, 2010.

[8] Liu, M. B. \& G. R. Liu. Smoothed Particle Hydrodynamics (SPH): an Overview and Recent Developments. Archives of Computational Methods in Engineering, 17: 25-76, 2010.

[9] Lucy, L. B. Numerical approach to testing the fission hypothesis. Astronomical Journal, 82: 1013-1024, 1977.

[10] Liu, M. B., Liu, G. R. \& Lam K. Y. Constructing smoothing functions in smoothed particle hydrodynamics with applications. Journal of Computational and Applied Mathematics, 155: 263-284, 2003.

[11] Morris, J. P., Fox, P. J. \& Zhu, Y. Modeling Low Reynolds Number Incompressible Flows Using SPH. Journal of Computational Physics, 136: 214-226, 1997.

[12] Monaghan J.J., Simulating free surface flow with SPH. Journal of Computational Physics, 110: 399-406, 1994.

[13] Pletcher, R. H., Tanehill, J. C. \& Anderson D. A. Computational Fluid Mechanics and Heat Transfer. CRC Press, $3^{\text {rd }}$ Edition, USA, 2013. 